\title{
EVALUACIÓN DEL SERVICIO AMBIENTAL HÍDRICO EN LA CUENCA DEL RÍO TEMPISQUE (COSTA RICA) Y SU APLICACIÓN AL AJUSTE DE TARIFAS
}

Gerardo Barrantes Moreno ${ }^{1}$

\begin{abstract}
Resumen
El estudio abordó la evaluación del servicio ambiental hídrico mediante la construcción de un modelo analítico que combina los aspectos biofísico y económico. En el caso biofísico se determinó el presupuesto hídrico y en el análisis económico se utilizaron tres conceptos de valor: de captación, de restauración y del agua como insumo de la producción. Estos valores orientan las posibilidades de un proceso de ajuste ambiental en las tarifas y eliminar así el subsidio ambiental que ha existido hasta ahora. Con el ajuste ambiental de tarifas se pretende generar recursos financieros para promover la conservación y restauración de ecosistemas mediante el mecanismo de Pago por Servicio Ambiental Hídrico, con el fin de favorecer la conservación del recurso hídrico y una gestión óptima del agua en la región, de tal manera que se avance hacia un manejo integral del recurso.
\end{abstract}

\section{Palabras clave}

Recursos hídricos, evaluación ambiental, ajuste de tarifas, servicio ambiental

\begin{abstract}
The study addressed the evaluation of environmental water service by building an analytical model that combines biophysical and economic aspects. In the biophysical case, the hydric budget was determined and in the economic analysis, three concepts of value were used: value of capture, value of restoration and value of water as a production input. These values guide the possibilities of a process of environmental adjustment in rates, and thus eliminate the environmental subsidy that has existed until now. With the environmental setting of fees, it is intended to generate financial resources to promote the conservation and restoration of ecosystems through the mechanism of payment for Hydric environmental services, to promote water conservation and optimum management of water in the region, so that it goes forward towards integrated water resources management. The development of a model is fundamental.
\end{abstract}

\section{Keywords}

Water resources, Environmental Assessment, fee adjustment, environmental service

1 Máster en Política Económica: énfasis Desarrollo Sostenible y Economía Ecológica. Director general y fundador del Instituto de Políticas para la Sostenibilidad (IPS). Correo E: gerardo@ips.or.cr 


\section{Introducción}

El recurso hídrico es motivo de preocupación a todo nivel de la sociedad, tanto en el ámbito nacional como internacional. Su escasez relativa amenaza con el desarrollo potencial al que podría aspirar una región $\mathrm{y}$, por tanto, esta escasez se convierte en una limitante importante para que la población mantenga o mejore su bienestar. Esta preocupación se acrecienta por la multiplicidad de factores y sus relaciones que explican el deterioro del recurso hídrico tanto en calidad como en cantidad.

El desafío social fundamental es conciliar los objetivos de desarrollo con los de conservación, para procurar el mantenimiento del equilibrio ecológico, actualmente debilitado y frágil. Esto es fundamental para mantener las funciones de los ecosistemas, de modo que se mantenga o mejore el flujo de bienes y servicios ambientales a la población, particularmente el flujo del servicio ambiental hídrico. Este esfuerzo, además de mejorar en calidad y cantidad las oportunidades de desarrollo, minimiza el riesgo inminente al que se ha sometido todo el aparato productivo debido a la escasez creciente de recursos naturales y específicamente del agua.

Un caso particular de interés es la Cuenca del Río Tempisque en Guanacaste, Costa Rica, donde se han identificado serios problemas de deterioro del recurso hídrico, con evidencias importantes de contaminación de aguas y agotamiento de acuíferos, al punto de que ya se empiezan a limitar los permisos para la explotación de aguas. Esto último, y bajo el modelo de crecimiento económico actual, parece indicar que las posibilidades de desarrollo de la cuenca se han alcanzado o rebasado, lo que genera una alarmante preocupación dado los niveles crecientes de población y de desarrollo de actividades productivas y, por lo tanto, de mayores necesidades de agua.

Una de las principales causas de esta problemática es que las tarifas de agua consideran sólo el costo financiero de brindar el servicio de abastecimiento, sin incluir los costos ambientales en que se debe incurrir para disponer de agua en calidad y cantidad socialmente aceptables. Esta debilidad tarifaria ha provocado el desperdicio de agua, el agotamiento de acuíferos y la degradación de grandes cuerpos de aguas superficiales, lo que ha puesto en alto riesgo tanto el bienestar de la población en general como la inversión económica instalada.

El servicio ambiental hídrico es uno de los principales mecanismos que se proponen para el ajuste correcto de tarifas y cánones por el aprovechamiento de agua, con el fin de fomentar el uso racional y las posibilidades de conservación de este recurso. La evaluación económica-ecológica de este servicio proporciona los montos monetarios para iniciar el proceso de ajuste de las tarifas de agua, de modo que se utilice el mecanismo de precios en la búsqueda de la optimización del recurso hídrico en sus distintos usos sociales. La idea es que los demandantes reconozcan a los oferentes un pago por los beneficios que les genera el disponer del servicio ambiental hídrico que ofrecen los ecosistemas de los cuales los segundos administran 
o son propietarios. Se parte del hecho que estos últimos asumen un costo financiero y de oportunidad al conservar sus recursos ecosistémicos.

\section{Servicios ambientales de los ecosistemas}

La forma en que la sociedad se beneficia de la disponibilidad de biodiversidad representa la transición conceptual de función ambiental a servicio ambiental, de modo que los flujos de los ecosistemas que entran, directa e indirectamente, al sistema social empiezan a reconocerse y a denominárseles servicios ambientales. Muchos de esos ecosistemas proporcionan productos y servicios económicos tradicionales tales como materias primas, frutos, y empleo, entre otros. Adicionalmente, se derivan de ellos otros servicios no tradicionales: captación de dióxido de carbono y su transformación en oxígeno, producción de material genético; medicamentos y materias primas para su producción; biodiversidad, protección del suelo contra la erosión, enriquecimiento de la calidad del suelo mediante los procesos de transformación de materiales orgánicos, protección de fuentes o mantos de agua, paisaje, belleza del paisaje y otros.

Recientemente, la escasez de los recursos naturales y la creciente demanda de servicios ambientales han aumentado el interés de la sociedad por identificar y valorar las funciones y los servicios ambientales que producen los ecosistemas, con el fin de fortalecer las decisiones de política. Por lo general, las políticas han sido débiles con respecto al aprovechamiento de los servicios ambientales del ecosistema, al punto que todavía no son reconocidos adecuadamente por el mercado, ni adecuadamente cuantificados para que se puedan comparar con servicios económicos y capital manufacturado (Bowers, 1997).

La sostenibilidad de la producción de servicios ambientales dependerá de la conservación de las existencias de activos naturales en cantidad y calidad. Si, a la vez, se reconoce que existen actividades económicas que compiten contra la protección del capital natural, entonces, el método del costo de oportunidad del uso de la tierra podría utilizarse para el cálculo del valor económico total, y el porcentaje asignado a cada uno de los componentes ya mencionados correspondería al valor económico del servicio ambiental.

\section{Importancia del bosque en la provisión del servicio ambiental hídrico}

La disponibilidad de agua es resultado de la capacidad que tienen los ecosistemas boscosos para captarla. Esta función es considerada un servicio ambiental del cual se beneficia la sociedad, tanto en la utilización productiva como en el consumo natural del recurso. Además, la disponibilidad de agua en los ecosistemas permite el 
desarrollo o presencia de otros bienes y servicios útiles para la sociedad. En el caso de disminución de tales ecosistemas por causas naturales o provocadas, repercute directamente en la regulación de los recursos hídricos y afecta el desarrollo de las diversas actividades humanas que sustentan: sistemas productivos agropecuarios, piscícolas, industriales, turísticos, generación hidroeléctrica y el suministro de agua potable a la población. También repercute sobre todos los ecosistemas relacionados con los recursos hídricos (Rudas, 1995). La disponibilidad del recurso hídrico en calidad y cantidad, además de ser un recurso vital, determina el potencial de crecimiento económico de una región o país (Reynolds, 1997; Azqueta, 1994).

Además, es de esperar que la remoción de la cobertura vegetal disminuya las posibilidades de infiltración lo que, a la vez, produce un incremento en la escorrentía durante los períodos lluviosos y afecta negativamente las posibilidades de almacenamiento de agua (Álvarez, 1995). En términos generales, en los bosques tropicales se da una relación directa entre la cobertura boscosa y los caudales: a mayor cobertura en bosque, mayores caudales. En tal sentido, es conveniente un proceso de conservación, protección y recuperación de cuencas. Por lo tanto, una mayor cobertura boscosa proporciona una mejor regulación de los recursos hídricos y disminuye los sedimentos que atentan con el mantenimiento de las infraestructuras desarrolladas para la producción de algún bien o servicio (CCT-CINTERPEDS, 1995; Calvo, 1990).

En términos generales, el bosque es un ente más eficiente en función de la calidad y la cantidad de agua, que cualquier otro ecosistema. De hecho, la presencia de bosques favorece la retención de agua, ya que el sistema radicular permite una mayor y mejor infiltración, y disminuye la escorrentía superficial (Ander, 1991). En un estudio realizado por CCT-CINTERPEDS (1995) se determinó que bajo cobertura de bosque la escorrentía es menor que bajo cobertura de pasto lo que justifica la mayor capacidad de infiltración del bosque. Además, se evaluó la calidad del agua y se determinó que bajo bosque había una calidad positiva de $81.44 \%$ y bajo pasto de $31.37 \%$.

La conversión de bosque a pasto u otros usos puede reducir drásticamente la capacidad de infiltración del suelo, dado que el volumen de recarga al subsuelo se favorece para aquellas áreas de la cuenca con mayor cobertura boscosa (Heuveldop et al., 1986). Según este autor, en un escenario de infiltración bajo tres tipos de cobertura: bosques, pastos y sin cobertura vegetal (suelo "desnudo"), el bosque tiene una mejor eficiencia en la infiltración $(68.92 \%)$, con relación al pasto y el suelo sin cubierta vegetal, los cuales presentan $24.75 \%$ y $6.33 \%$ de eficiencia, respectivamente.

\section{Datos generales de la cuenca del río Tempisque}

La cuenca del Río Tempisque se encuentra en el Pacífico Norte de Costa Rica, a unos $150 \mathrm{~km}$ al norte este de San José. La cuenca posee dos grandes bloques, uno 
del Río Bebedero que drena al Tempisque hacia la desembocadura y el otro bloque de los ríos Liberia, Ahogados y Tempisquito. En general, estos ríos se originan en las estribaciones de la Cordillera de Guanacaste que posee una elevación máxima de $1900 \mathrm{~m}$. La cuenca posee un área de $5,437.5 \mathrm{~km}^{2}$ lo que corresponde al $10.6 \%$ del área de Costa Rica, y posee una forma redondeada con una anchura máxima de este a oeste de $105 \mathrm{~km}$ y de norte a sur una distancia máxima de $93 \mathrm{~km}$.

En cuanto a uso de la tierra se destaca la notable disminución y fragmentación de los bosques, independientemente si son primarios o secundarios. La principal actividad económica que compite con el bosque por el uso del suelo en la cuenca es pasto (Mapa 1). El área de bosque representa un 38.7\%, mientras que en pasto está el $42.5 \%$ del área. La cuenca tiene conflicto de uso en un $23 \%$ del área (127,454ha) lo que significa que hay actividades en suelos que por sus condiciones no pueden soportar sin degradarse, debido a limitaciones por pendiente, erosión y profundidad.

Mapa 1. Uso y cobertura del suelo, Cuenca del Río Tempisque, 2000

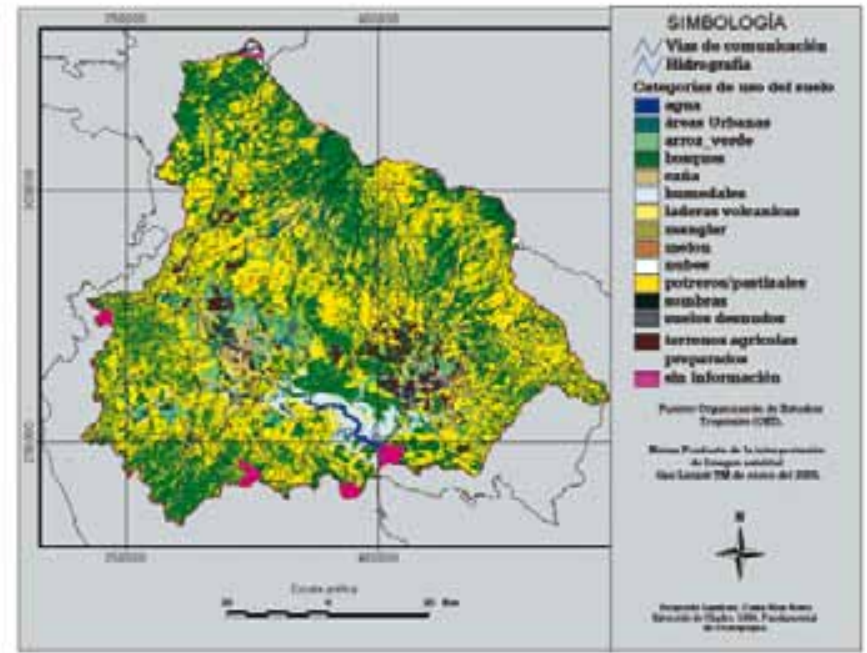

Fuente: OET. Organización de Estudios Tropicales.

Dado que la región se encuentra en la vertiente pacífica de Costa Rica, climáticamente se caracteriza por una estación seca prolongada de hasta seis meses (de diciembre a mayo). En la estación lluviosa se presentan importantes disminuciones de la precipitación a mediados del año. Cerca del $45 \%$ de la precipitación total se registra en los meses septiembre y octubre. La temperatura media anual para toda 
la superficie de la cuenca del Tempisque de $26.78^{\circ} \mathrm{C}$, y el rango de variación va de $\operatorname{los} 19.55^{\circ} \mathrm{C}$ en el sector de Monte Verde a los $29.05^{\circ} \mathrm{C}$, en el sector de Cañas y una elevación media de $220 \mathrm{msnm}$.

\section{Presupuesto hídrico en la cuenca del río Tempisque}

El presupuesto de aguas se refiere a la cuantificación física de la oferta y la demanda de agua en una región, con el fin de generar información relacionada con las interacciones recurso-usuarios que permita una mejor administración del recurso. Para la construcción del presupuesto de aguas se requiere de la comprensión del ciclo hidrológico ${ }^{2}$-relación atmósfera tierra- y del ciclo hidrosocial -administración social del recurso-, como dos grandes componentes que determinan la disponibilidad y el aprovechamiento del recurso hídrico. El ciclo hidrológico explica, fundamentalmente, el proceso natural que mantiene una oferta de agua y el ciclo hidrosocial explica la forma en cómo se administra la demanda de agua en el ámbito económico.

\subsection{Modelo analítico para determinar el presupuesto hídrico}

\subsubsection{Oferta hídrica}

La oferta total de agua de una unidad de análisis está dada por la precipitación, principalmente. Sin embargo, en caso de ser necesario se deben analizar aportes de otras cuencas por obras de riego o drenaje. El cálculo correspondiente a la oferta total se obtiene mediante la ecuación:

$$
O T=\sum_{i=1}^{n} 0.001 \bar{P}_{i} * A_{i}+O e
$$

Donde,

OT Oferta total hídrica en el área de importancia ( $\left.\mathrm{m}^{3} / \mathrm{año}\right)$

$\overline{P i} \quad$ Precipitación media en la cuenca i (mm/año)

$n$ Número de cuencas

$\mathrm{A}_{\mathrm{i}} \quad$ Área de la cuenca $\mathrm{i}\left(\mathrm{m}^{2}\right)$

$\mathrm{O}_{\mathrm{e}} \quad$ Aporte hídrico externo a la cuenca ( $\left.\mathrm{m}^{3} / \mathrm{año}\right)$

Nota: $1 \mathrm{~mm}=0.001 \mathrm{~m}^{3} / \mathrm{m}^{2} / \mathrm{año}$, este es un factor de conversión que se utiliza para uniformizar unidades para el presupuesto hídrico.

2 El ciclo hidrológico muestra los estados, procesos y magnitudes de cada uno de los componentes que caracterizan el movimiento del agua desde los océanos hasta la atmósfera, la tierra y de vuelta a los océanos; y, por tanto, constituye el marco conceptual para analizar la interacción suelo-agua-vegetación. 
De la oferta total, un porcentaje regresa a la atmósfera a través del proceso de evapotranspiración, queda potencialmente disponible sólo una parte de ella para el abastecimiento de las distintas actividades económicas y poblacionales. La estimación de la oferta disponible para la satisfacción de la demanda social está dada por la siguiente ecuación:

$$
O d=\sum_{i=1}^{n}\left(O T_{i}-0.001 E T_{i} * A_{i}\right)
$$

Donde,

Od Oferta hídrica disponible en el área de importancia ( $\left.\mathrm{m}^{3} / \mathrm{año}\right)$

ETi Evapotranspiración en el área de importancia hídrica

en la cuenca i (mm/año)

\subsubsection{Recarga potencial}

Por otro lado, la infiltración es la capacidad del terreno para aceptar agua, y de poder transmitirla a horizontes inferiores. La infiltración está controlada por varios factores, entre ellos, el tipo en intensidad de las lluvias, la pendiente, la textura del suelo y la vegetación. Para determinar la infiltración potencial se aplica el modelo analítico propuesto por Schosinsky \& Losilla (2000). Con esta propuesta se pueden delimitar las áreas potenciales de recarga de los acuíferos a nivel regional. La infiltración potencial está determinada según el modelo propuesto por Losilla y Schosinsky (2000) como:

$$
I p=\Sigma 0.001(1-\mu)(K p+K v+K f c) * \overline{P i} * \mathrm{Ai}
$$

Donde,

Ip infiltración potencial ( $\left.\mathrm{m}^{3} / \mathrm{año}\right)$

$K p \quad$ fracción que infiltra por efecto de la pendiente

$K v$ fracción que infiltra por efecto de la cobertura vegetal

$K_{f c}$ fracción que infiltra por efecto de textura del suelo

$\mu \quad$ fracción de la precipitación que no llega al suelo por la intercepción de copas.

La recarga potencial $(\mathrm{R})$ o infiltración neta estará dada por: 


$$
R=I p-\left(E_{2}+T\right)
$$

Donde,

$E_{2}$ evaporación $\left(\mathrm{m}^{3} / \mathrm{año}\right)$

$T$ transpiración $\left(\mathrm{m}^{3} / \mathrm{año}\right)$

$R \quad$ recarga potencial ( $\left.\mathrm{m}^{3} / \mathrm{año}\right)$

Mientras que la escorrentía superficial (ESCs $\left.\mathrm{m}^{3} / \mathrm{año}\right)$ está dada por:

$$
E S C s=O d-R
$$

\subsubsection{Demanda hídrica}

En términos generales se puede agrupar la demanda total en demanda natural explicada por la evapotranspiración real y la demanda social por el consumo de agua en los distintos sectores de la economía y las fugas ocasionadas en el proceso de aprovechamiento de las aguas por los distintos sectores. La ecuación siguiente se propone para la estimación de la demanda social.

$$
Q^{s}=\sum_{i=1}^{n} \sum_{j=1}^{m} q_{i j}+\sum_{i=1}^{n} f_{i}
$$

Donde,

$Q^{s} \quad$ Demanda social hídrica en la cuenca ( $\left.\mathrm{m}^{3} / \mathrm{año}\right)$

$q_{i j} \quad$ Volumen de agua demandada por el sector $\mathrm{j}$ en la cuenca i ( $\left.\mathrm{m}^{3} / \mathrm{año}\right)$

$f_{i} \quad$ Volumen de agua perdido por fugas en la cuenca i ( $\left.\mathrm{m}^{3} / \mathrm{año}\right)$

Por lo tanto, el cálculo de la demanda total se puede determinar mediante la siguiente ecuación:

$$
D T=E T_{r}+Q^{s}
$$

Donde,

DT Demanda total hídrica en la cuenca ( $\left.\mathrm{m}^{3} / \mathrm{año}\right)$

\subsubsection{Estimaciones del presupuesto hídrico}

Con los datos promedios anuales de 52 estaciones meteorológicas provenientes de diferentes fuentes (IMN, ICE, OET) se elaboró un modelo de precipitación media 
anual en celdas de $15 \mathrm{~m} * 15 \mathrm{~m}$ para la superficie total de la cuenca. La precipitación media es de $1,738.97 \mathrm{~mm} /$ año $\left(9,455.62 \mathrm{mill} . \mathrm{m}^{3} /\right.$ año de agua), con una desviación estándar de $425.15 \mathrm{~mm}$, un mínimo de 518.51 y un máximo de 5,280.3mm anuales. La evapotranspiración real media es de $1,116.44 \mathrm{~mm}$ /año con un mínimo de $554.22 \mathrm{~mm}$ y un máximo de $1482.95 \mathrm{~mm}$, lo que equivale al $64.2 \%$ de la precipitación media en la cuenca.

El balance hídrico (Mapa 2) implica que la escorrentía total (superficial e infiltración) es de $622.58 \mathrm{~mm} / \mathrm{año}$, que resulta de la diferencia entre la precipitación media y la evapotranspiración real. Su distribución refleja situaciones críticas para algunas regiones de la cuenca, donde se presenta déficit; es decir, la evapotranspiración es superior a la precipitación. En el mapa se observa que las zonas de mayor importancia están ubicadas en la cordillera volcánica de Guanacaste, donde se presentan las mayores precipitaciones de la cuenca y que resultan importantes de proteger. La mayor parte de la cuenca presenta una oferta disponible (escorrentía total) entre 465 y $1431 \mathrm{~mm}$. Hay que añadir a la oferta natural disponible el aporte que se da por el Distrito de Riego Arenal-Tempisque (DRAT) cuya agua proviene de la cuenca del Arenal, fuera de la cuenca del río Tempisque.

Mapa 2. Balance hídrico, Cuenca Río Tempisque, Guanacaste, 2001

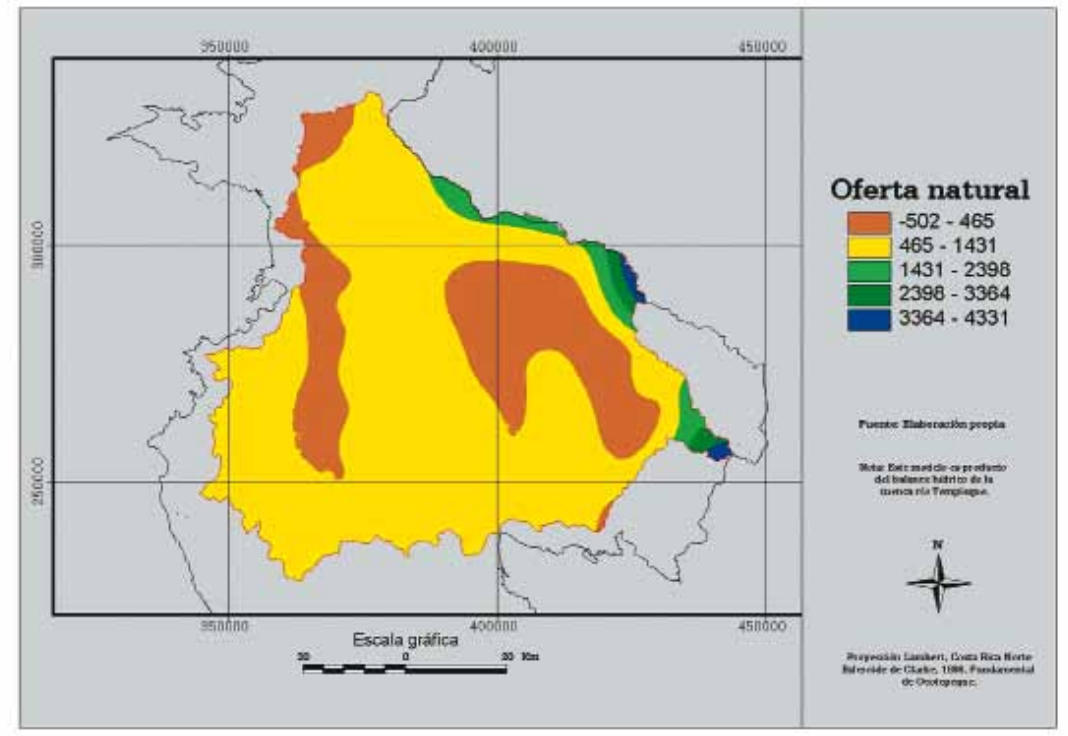

Fuente: Elaboración propia 
Se hizo un análisis ${ }^{3}$ del potencial de recarga acuífera y su distribución en la cuenca, encontrándose una gran fracción de la cuenca donde dicha recarga potencial es negativa (Mapa 3). Es decir, que la cuenca aporta al proceso de evapotranspiración en los sitios donde la precipitación es menor que la evapotranspiración. El promedio estimado para la recarga potencial es de $144.2 \mathrm{~mm} / \mathrm{año}$, lo que representa el $8.3 \%$ de la precipitación promedio total. Las zonas identificadas de mayor potencial de recarga deben ser consideradas prioritarias para iniciar un modelo de aprovechamiento del suelo que no amenace dicho potencial, ya sea que lo limite en cantidad o sea afectado en calidad por contaminación. También, el potencial de recarga puede ser considerado como un criterio prioritario para la asignación de pago por servicio ambiental hídrico.

Mapa 3. Recarga esperada en la cuenca del Río Tempisque

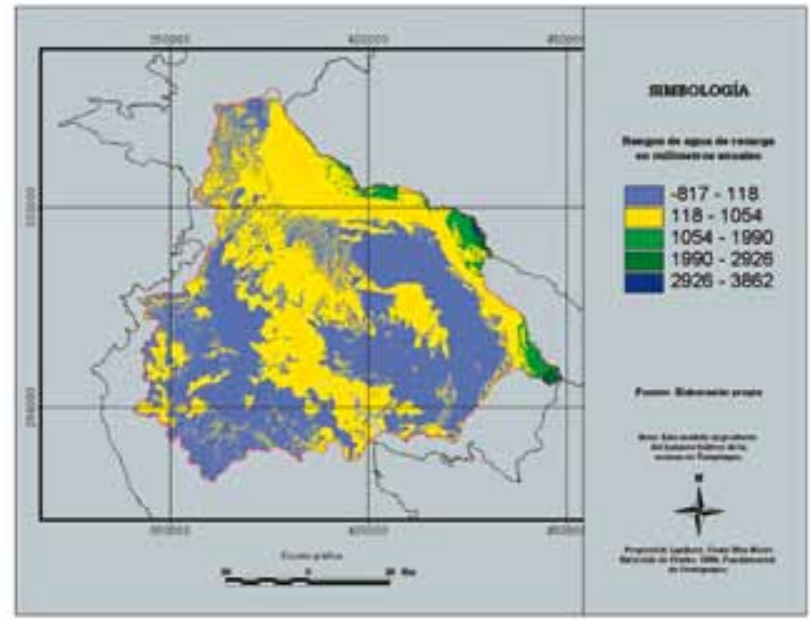

Fuente: Elaboración propia

De acuerdo con la información del Departamento de Aguas del Ministerio de Ambiente y Energía (MINAE), del Servicio Nacional de Riego y Avenamiento (SENARA) y de Acueductos y Alcantarillados (A y A), la demanda total es de 4,782.96 millones de metros cúbicos, de los cuales el $98.37 \%$ representa agua

3 El análisis está basado en la metodología de Schosinsky y Losilla (2000). Para la estimación de la recarga se consideró que $12 \%$ de la precipitación es interpretada por el follaje y que se evapora, mientras que el $88 \%$ restante un $66 \%$ se espera que un filtre de acuerdo con la metodología de Schosinsky y Losilla (2000). De este $66 \%$ una parte es demandada por el sistema natural a través de la evaporación y la transpiración de las plantas y la otra parte se espera que recargue. 
superficial. Los 77.66 millones de metros cúbicos anuales demandados en agua subterránea corresponde a 111 pozos $(8.35 \%)$ de los 1330 que están registrados en SENARA. Bajo estas circunstancias la estimación del consumo de agua subterránea se considera una subestimación que debe ser atendida en un proceso de mejoramiento en los controles y monitoreo de concesiones por parte del Departamento de Aguas. A la anterior preocupación, hay que añadir que hay una cantidad importante de pozos que no están ni siquiera registrados en SENARA, por lo que la subestimación se hace más acentuada.

La situación actual de la cuenca del Río Tempisque, sin considerar la preocupación anterior, presenta un excedente total estimado en $303.06 \mathrm{mill} . \mathrm{m}^{3} / \mathrm{año}$, explicado fundamentalmente por la subestimación en la demanda de agua subterránea. Tal y como está en el cuadro, hay una demanda social de agua superficial que excede la oferta disponible en $377.51 \mathrm{mill} . \mathrm{m}^{3} / \mathrm{año}$, lo que hace pensar que se sobreutiliza este recurso; es decir, se consumen las reservas, dado que el consumo supera el flujo anual hídrico por precipitación natural.

Cuadro 1. Presupuesto hídrico para la cuenca del Río Tempisque

\begin{tabular}{lr}
\hline & $\begin{array}{c}\text { Volumen } \\
\text { mill.m }{ }^{3} \text { año }\end{array}$ \\
\hline Oferta & \\
Total $^{*}$ & $\mathbf{1 0 , 9 9 4 . 4}$ \\
Disponible & $5,086.02$ \\
$\quad$ Agua superficial total & $4,327.79$ \\
$\quad$ Natural & $2,771.22$ \\
Importada DRAT & $1,556.56$ \\
Recarga esperada & 758.24 \\
Demanda & \\
Total $\quad$ Evapotranspiración & $\mathbf{1 0 , 9 9 4 . 4}$ \\
Actividades humanas & $5,908.40$ \\
Agua superficial & $4,782.96$ \\
Agua subterránea & $4,705.30$ \\
Excedente disponible & 77.66 \\
Agua superficial & 303.06 \\
Agua subterránea & $\mathbf{- 3 7 7 . 5 1}$ \\
& 680.57 \\
\hline
\end{tabular}

* Comprende la precipitación y el aporte que se da desde la represa del Arenal

Fuente: Elaboración propia 


\section{Valoración económica del servicio ambiental hídrico en la cuenca del Río Tempisque}

La valoración económica del servicio ambiental hídrico se basa en tres componentes principales. El primero se refiere al valor del bosque en su función de regulación del flujo hídrico, el cual llamamos valor de captación. El segundo está asociado con la restauración de cobertura boscosa o de ecosistemas que han sido degradados y que son de alta importancia para la conservación del recurso hídrico, el cual se denominó valor de restauración. El tercero tiene relación con el valor de uso del agua en las distintas actividades económicas en las que sirve como insumo de la producción, y que se denomina valor del agua como insumo de la producción. Hay un último valor que responde más a un aspecto de previsión financiera ante eventualidades ocasionadas por eventos meteorológicos que deben ser atendidas oportunamente, el que se ha denominado margen de ahorro-inversión.

\subsection{Valor de captación}

La productividad del bosque en el caso del servicio ambiental hídrico, está determinada por la cantidad de agua captada anualmente, $\mathrm{y}$, su valor económico estará asociado con la actividad económica que compite con el bosque. Sólo se justifica, bajo la concepción de la economía de los recursos naturales, la transformación del uso del suelo de bosque natural a otros usos, si los ingresos anuales por los otros usos superan los ingresos anuales por servicios ambientales generados por el bosque. En este sentido, una hectárea de bosque se protegerá, cuando el valor de sus servicios ambientales se equipare con el costo de oportunidad de los demás usos del suelo. Así, la recuperación y conservación de los bosques existentes se fundamenta, en parte, en su importancia económica por los servicios ambientales que ofrece.

Por lo anterior, el costo de oportunidad es una metodología válida para valorar económicamente el componente de captación hídrica del bosque y de otros servicios ambientales de importancia económica reconocida. Esta valoración obedece a la necesidad de tener un indicador económico de la productividad del bosque que debe ser compensada por la sociedad, para que el dueño de la tierra considere al bosque como una actividad económica tan rentable como la que se deja de realizar, y se convierta así en un productor de servicios ambientales reconocidos y pagados por la sociedad (Castro y Barrantes, 1998).

Para estimar el valor de captación como un componente que determina la productividad hídrica del bosque, se necesita:

- El volumen anual de agua captada y fijada por los bosques en las zonas de recarga de la cuenca. 
- Cálculo del costo de oportunidad del uso de la tierra en esas zonas.

- Ponderación de la importancia del bosque en términos de su productividad hídrica, al compararla con los otros servicios de la biodiversidad.

Además, es necesario considerar el efecto positivo que tiene el bosque sobre la calidad del agua de escorrentía superficial. La Ecuación 1 permite estimar el valor de captación del bosque:

$$
V C=\sum_{i=1}^{n} \frac{\alpha_{i} B_{i} A b_{i}}{O c_{i}}
$$

Donde,

VC Valor de captación hídrica del bosque $\left(\varnothing / \mathrm{m}^{3}\right)$

$\mathrm{B}_{\mathrm{i}} \quad$ Costo de oportunidad de la actividad económica que compite con el bosque por el uso del suelo en la cuenca i ( $\notin /$ ha/año)

$\mathrm{Ab}_{\mathrm{i}}$ Área bajo bosque en la cuenca i (ha)

$\mathrm{Oc}_{i}$ Volumen de agua captada en la cuenca i ( $\left.\mathrm{m}^{3} / \mathrm{año}\right)$

$\alpha_{i} \quad$ Importancia del bosque en la cuenca i en función de la cantidad y calidad del recurso hídrico $0 \leq \alpha \leq 1$.

n Número de cuencas involucradas

En la cuenca del Río Tempisque hay 210.226,8ha. de bosque de acuerdo con el mapa de uso y cobertura del 2000 (Mapa 1). La siguiente cobertura en importancia es la de pasto para la actividad ganadera, lo que indica que esta es la actividad que compite con la disponibilidad de bosque. La superficie boscosa deberá ser compensada por el costo de oportunidad que significa el no usarla en ganadería $\mathrm{y}$, por lo tanto, esta compensación es la que garantizaría la posibilidad de que se mantenga el uso del suelo bajo bosque. Esta es la superficie que será considerada en la estimación del valor de captación hídrica, dado que los esfuerzos de conservación estarán orientados a mantener esta disponibilidad de bosque.

La estimación del costo de oportunidad se basó en los beneficios netos de la actividad ganadera. Con base en el estudio la Agencia de Cooperación Internacional del Japón (2002) el costo de la ganadería se estimó en $\not 181.810 /$ ha/año, donde se considera el período de engorde de 12 meses para un animal de $450 \mathrm{~kg}$. Por otro lado, el precio estimado varía de $\notin 370 / \mathrm{Kg}$. a $\varnothing 500 / \mathrm{kg}$. Considerando el precio de $\varnothing 500 /$ 
$\mathrm{kg}$ se determina que el ingreso esperado es de $\phi 225.000 / \mathrm{ha} / \mathrm{año}$, lo que genera un beneficio de $ф 43190 /$ ha/año. Se usará este valor como costo de oportunidad en el cálculo del valor de captación hídrica del bosque. A este costo habría que añadir el costo respectivo a la administración y atención del bosque, cuyo monto es de $\not 13,617 /$ ha/año de acuerdo a un estudio de CATIE (1996) que lo estimó en programas de reforestación en el quinto año y que se podría considerar constante para el resto de los años.

Dado que el costo de oportunidad debe ser cubierto por los distintos usuarios de los servicios que brindan los ecosistemas boscosos, es necesario estimar la importancia que tiene este recurso para la conservación del agua. Esta importancia se estimó con base en una encuesta, cuyo resultado señala que la importancia hídrica del bosque es de un $44.02 \%$. El restante $55.98 \%$ se distribuye en los demás servicios ambientales por los cuales resulta importante conservar bosque. Si se ajusta la estimación de la importancia a los servicios ambientales que se establecen en la legislación (fijación de gases, protección hídrica, protección de la biodiversidad y belleza paisajística), la proporción anterior se distribuye en un $49.22 \%$ para la conservación hídrica y un $50.78 \%$ para los otros tres servicios ambientales en conjunto.

Al considerar la importancia hídrica del bosque en un $49.22 \%$, la proporción del costo de oportunidad que debe ser compensado por los usuarios del agua a los propietarios de tierra involucrados en protección de bosques, equivale a $₫ 27,960.4 /$ ha/año. De esta manera, al tomar la cobertura boscosa y la oferta hídrica disponible en la cuenca, se determina un valor de captación de $\not 1.67 / \mathrm{m}^{3}$.

\subsection{El valor de restauración}

La restauración de bosques en cuencas degradadas es un mecanismo que ayuda a la conservación de las aguas superficiales y subterráneas y evita la erosión de los suelos (Ramakrisna, 1997). Estos beneficios llevan implícito un costo que ha de considerarse dentro de la estructura de valoración económico-ecológica para el uso del agua, con el fin de proporcionar recursos financieros para el desarrollo de actividades orientadas a la protección, recuperación y conservación de las partes altas de las cuencas (Castro y Barrantes, 1998).

Los costos incurridos en la restauración de bosques se determinan por los gastos en salarios, cargas sociales de personal destinado a la protección, gastos en combustible, transporte, infraestructura y otros gastos de operación e incentivos utilizados para la protección ambiental, otros desembolsos necesarios para el sostenimiento del capital natural existente en laderas. De acuerdo con las características del bosque natural, el costo de restablecimiento debería ser equivalente al de recuperar el ecosistema para dejarlo en condiciones similares a las que éste mantenía antes de ser intervenido (United Nations, 1993). Esos costos no están estrictamente en función del recurso hídrico, por lo que habrá que asignar una ponderación del total de esos costos que se asocian con la protección del recurso hídrico, lo cual requiere: 
- Cálculo del número de hectáreas que deben ser recuperadas

- Cálculo de los costos de restauración considerada una situación similar a la del bosque natural antes de ser degradado

- Ponderación de la importancia del bosque en términos de su productividad hídrica

- Volumen hídrico captado en la cuenca.

Por lo tanto, en términos operacionales se puede plantear que los recursos necesarios para el establecimiento de las medidas de recuperación, protección, conservación y mantenimiento de cuencas, están dados por la Ecuación 2.

$$
V P=\sum_{i=1}^{n} \sum_{j=1}^{m} \frac{\delta_{i j} C_{i j} A r_{i}}{O c_{i}}
$$

Donde,

VP Costo de restauración de bosques en cuencas hidrográficas $\left(\not / \mathrm{m}^{3}\right)$

$\mathrm{C}_{\mathrm{ij}} \quad$ Costos para la actividad $\mathrm{j}$ destinada a la restauración del bosque en la cuenca i ( $\varnothing /$ ha/año)

$\mathrm{Ar}_{\mathrm{i}}$ Área a restaurar en la cuenca i (ha)

$\delta_{j} \quad$ Fracción del costo j destinado a la restauración del bosque en función del recurso hídrico en la cuenca i (\%)

m Número de insumos utilizados

n Número de cuencas involucradas

En la cuenca del Río Tempisque hay 127,457ha en conflicto de uso del suelo, de acuerdo con su capacidad de uso. Bajo este criterio, esta es la superficie a recuperar de manera prioritaria con el fin de mejorar el régimen hídrico en la cuenca y, con ello, la disponibilidad de agua. El mejoramiento de estas condiciones disminuye el riesgo de la inversión establecida y favorece una mayor capacidad de desarrollo para la Región Chorotega que por escasez de agua enfrenta serios problemas productivos y sociales que hacen ver que Guanacaste ha encontrado un techo a su desarrollo. 
El valor de restauración de la cuenca del Río Tempisque, está asociado con los costos de desarrollar las distintas actividades requeridas para lograrlo. Estos costos comprenden todos los gastos anuales que deben realizarse en salarios, reforestación, gastos administrativos, gastos de mantenimiento en infraestructura y equipo, y otros gastos asociados. Asumido un período de cinco años para llegar a tener un sistema de restauración relativamente consolidado, el costo total es de $\phi 262,191.82 /$ ha./año para el Pacífico seco (CATIE, 1996). De estos costos totales, el 48.28\% se invierte el primer año de operación del sistema, a partir del cual se reduce hasta llegar a un monto relativamente fijo para los años del cuarto en adelante, ya que se asocian con costos de mantenimiento solamente.

Habida cuenta de la capacidad hídrica en la cuenca, la importancia hídrica del bosque en $49.22 \%$ y la superficie en conflicto de uso del suelo, así como los costos de restauración promedio para el primer año equivalentes a $\phi 126,580 /$ ha y la rentabilidad de la actividad ganadera estimada en $\notin 43,190 /$ ha/año, se obtiene un valor de restauración de $\notin 3.02 / \mathrm{m}^{3}$. Dicho valor debe mostrar un comportamiento de disminución en los siguientes años, debido a una disminución en los costos de reforestación. Después de los cinco años, el costo tiende a mantenerse constante, relacionándose, fundamentalmente, con los costos de mantenimiento de las áreas reforestadas. Por lo tanto, a partir del quinto año, se debe mantener un valor de restauración constante en el modelo tarifario por un período previamente definido, a partir del cual se inicie el pago del valor de captación en sustitución del de restauración.

\subsection{Valor del agua como insumo de la producción en la cuenca del Río Tempisque}

El agua es un insumo más en los procesos productivos de las distintas actividades económicas, por lo cual debe contar con un precio que refleje su escasez relativa. La estimación de este precio se hace de acuerdo con los usos domésticos e industrial para los cuales se usó el enfoque de excedente del consumidor; el uso agrícola donde se usó el cambio en productividad anual y el uso en hidroenergía donde se usó el enfoque de ahorro en costos.

\subsubsection{Valor del agua en el sector doméstico e industrial}

La aproximación del valor económico del agua, se basa en los beneficios sociales que se derivan cuando hay un cambio en la oferta total del recurso, debido fundamentalmente a un incremento en la demanda, ya sea por el crecimiento poblacional o por el desarrollo económico. La aplicación del enfoque de excedente del consumidor, considerado el análisis de demanda de agua, reporta un valor económico asociado con incrementos en la oferta de agua y cómo dichos incrementos 
corresponden a variaciones netas de utilidad para la población (Ferreiro, 1994). Para la aplicación de este enfoque, es necesario conocer la elasticidad precio de la demanda, con el fin de obtener la disponibilidad de pago del consumidor del recurso hídrico en los sectores doméstico e industrial.

El valor del agua en los sectores doméstico e industrial, puede estimarse mediante el análisis de demanda (excedente del consumidor), donde se incluyen variables como precio (tarifa), volumen consumido y elasticidad ${ }^{4}$. Cuando algún factor determinante de la demanda (p.e. el crecimiento poblacional) hace que ésta se desplace de $\mathrm{D}_{1}$ a $\mathrm{D}_{2}$ $\mathrm{y}$, considerado constante el precio $\mathrm{p}_{1}$ inicial, el valor neto o excedente de la población por el incremento en la oferta de agua será el área $A B C=B C Q_{1} Q_{2}-A B Q_{1} Q_{2}(G r a ́ f i c o$ 1). Ese excedente $A B C$ representa la valoración social neta del incremento de la oferta de $\mathrm{Q}_{1}$ a $\mathrm{Q}_{2}$. Esta cantidad neta corresponde al valor del agua en el origen, antes de ser captada, transportada, potabilizada y distribuida.

Gráfico 1. Curvas de demanda para el análisis del valor económico del agua

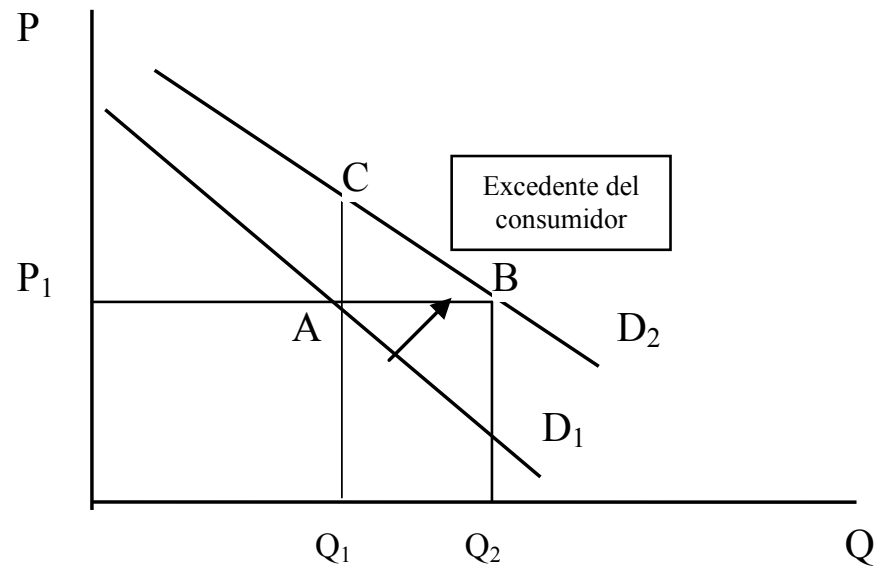

Fuente: Adaptado de Azqueta y Ferreiro, 1994.

Se puede aproximar una curva de demanda al tomar un par inicial precio-cantidad y suponer una elasticidad precio constante para la demanda. La curva de demanda puede suponerse una función Cobb-Douglas que puede expresarse mediante la ecuación:

$4 \quad$ Elasticidad se refiere a la respuesta del consumidor ante cambios en los precios. La elasticidad puede obtenerse con los datos sobre precio y consumo, o bien adoptar un parámetro estimado para otra población con características similares. 


$$
Q=k P^{\varepsilon}
$$

Donde,

Q Volumen de agua $\left(\mathrm{m}^{3} / \mathrm{mes}\right)$

P Tarifa financiera actual por el servicio de abastecimiento del agua $\left(\phi / \mathrm{m}^{3}\right)$

k Factor de proporcionalidad

$\varepsilon \quad$ Elasticidad precio de la demanda

En el caso de tener información sobre un par de puntos $\left(\mathrm{Q}_{1}, \mathrm{P}_{1}\right)$ y sobre la elasticidad $\varepsilon$, se calcula $\mathrm{k}_{1}$ para la curva de demanda del período $1\left(\mathrm{D}_{1}\right)$ :

$$
\mathrm{k}_{1}=\mathrm{Q}_{1} \mathrm{P}_{1}^{-\varepsilon}
$$

Así como se obtiene $\mathrm{D}_{1}$ se obtienen las curvas de demanda de otros períodos, postulado un desplazamiento de dichas curvas conforme a una tasa de crecimiento $\mathrm{r}$ (en función del crecimiento poblacional y/o de la renta), tal que $\mathrm{k}_{\mathrm{t}}=\mathrm{k}_{1}(1+\mathrm{r})^{\mathrm{t}-1}$ donde $\mathrm{t}$ representa los períodos futuros.

Estimados $\mathrm{D}_{1}$ y $\mathrm{D}_{2}$ para dos períodos cualesquiera y supuesto que el aumento en la oferta esté acompañado de un incremento en el precio (porque el coste medio del abastecimiento adicional sea superior al abastecimiento inicial), el valor neto del agua, VA, del excedente social atribuible al incremento en el consumo de agua aplicable a cada sector (doméstico y industrial), vendría dado por:

$$
V A=\frac{P_{1}\left(Q_{2}^{\frac{1}{\varepsilon}+1}-Q_{1}^{\frac{1}{s}+1}\right)}{Q_{1}^{\frac{1}{\varepsilon}}\left(\frac{1}{\varepsilon}+1\right)}-P_{2}\left(Q_{2}-Q_{1}\right)
$$

Donde, $\mathrm{P}_{2}\left(\mathrm{Q}_{2}-\mathrm{Q}_{1}\right)$ representa el costo social del abastecimiento adicional de agua.

En el caso del sector doméstico, por lo general, el agua es para consumo humano y para los quehaceres básicos de la familia. Sin embargo, a cierto nivel de demanda el agua es usada por las familias para otros fines como piscinas, riego de jardines y lavado de carro, entre otros. Es por eso, que resulta justificable la asignación de un precio para el agua como insumo de la producción en el sector doméstico después de cierto nivel de consumo. 
Para la estimación del valor del agua en el sector doméstico, se consideró la demanda, la tarifa y un nivel de elasticidad ${ }^{5}$ precio de la demanda de -0.25 . Además, se consideró una tasa de crecimiento $2.9 \%$ para el sector doméstico en la Región Chorotega. La estimación se realizó como un promedio ponderado que considera zona rural y la urbana y equivale a $ф 3.57 / \mathrm{m}^{3}$.

En cuanto a la industria el agua es usada para la producción de otros bienes y servicios que tienen un precio en el mercado. Para la estimación del valor del agua en estos sectores se consideró la demanda y la tarifa existentes en A y A, la elasticidad precio-demanda $-0.30 \%$ y una tasa de crecimiento de estos sectores de $3.5 \%$. La estimación se realizó como promedio ponderado que incluye la zona rural y la urbana, obteniéndose $\notin 13.67 / \mathrm{m}^{3}$.

\subsubsection{Valor del agua en el sector hidroenergía}

Con respecto al enfoque del ahorro en costos, su aplicación permite cuantificar el monto que el país se ahorra con hidroelectricidad en comparación con cualquier otra alternativa de generación eléctrica, incluida la importación de energía, en el abastecimiento de la demanda nacional de electricidad. Este ahorro corresponde al valor económico que se le puede asignar al agua en el sector hidroeléctrico, cuando ésta es considerada como insumo de la producción. Para aplicar el ahorro en costo debido a la utilización del agua en la generación de electricidad, es necesario contar con los precios de todas las demás opciones que puede usar el país para suplir la demanda nacional de electricidad, incluida la hidroeléctrica. De esta manera, se compara la mejor opción alternativa con la de hidroelectricidad y la diferencia proporciona el valor de la mejor opción económica (Castro y Barrantes, 1998).

Con los distintos precios para el abastecimiento de la demanda nacional de electricidad se puede estimar el ahorro que le significa al país, la utilización del agua en la producción de electricidad. El cálculo se basa en la siguiente fórmula:

$$
\mathrm{P}_{\text {aho }}^{\mathrm{kw}}=\mathrm{P}^{*}-\mathrm{Ph}^{\mathrm{h}}
$$

Donde,

$$
\begin{array}{ll}
\mathrm{P}^{\mathrm{kw}}{ }_{\text {aho }} & \text { Ahorro por } \mathrm{kW} \text { generado }(\not / \mathrm{kW}) \\
\mathrm{P}^{\mathrm{h}} & \text { Precios del } \mathrm{kW} \text { generado con hidroelectridad }(\not / \mathrm{kW}) \\
\mathrm{P}^{*} & \text { Precio de la siguiente mejor alternativa seleccionada }(\not / \mathrm{kW})
\end{array}
$$

5 La elasticidad fue tomada de informes de A y A sobre estudios de demanda en varias partes del país. La demanda de agua es inelástica con respecto al precio; es decir, por cada $1 \%$ de cambio en el precio, la demanda se reduce $0.30 \%$. 
La diferencia entre estos dos costos representaría el ahorro que significa para el país el abastecimiento de la demanda de electricidad con una planta hidroeléctrica. Conocida la cantidad de $\mathrm{kW}$ por metro cúbico que se genera y el monto ahorrado por cada $\mathrm{kW}$, el valor de un metro cúbico de agua sería:

$$
\mathrm{PE}_{\mathrm{ag}}=\mathrm{P}_{\text {aho }}^{\mathrm{kw}} * \mathrm{q}^{\mathrm{kw}}
$$

Donde,

$\mathrm{PE}_{\mathrm{ag}} \quad$ Precio del agua en el Sector de Electricidad $(\phi / \mathrm{kW})$

$\mathrm{q}^{\mathrm{kw}} \quad$ Cantidad de $\mathrm{kW}$ por $\mathrm{m}^{3}\left(\mathrm{~kW} / \mathrm{m}^{3}\right)$

El valor del agua en hidroenergía está basado en los costos que se ahorra el país por no producir con otras fuentes, como la térmica o la geotérmica. Para el cálculo correspondiente se consideró el costo de producción $(\Varangle / \mathrm{kWh})$ bajo distintas fuentes de producción de energía del ICE para el año 2000 (hidroenergía, térmica, geotérmica, eólica, importada). Según los datos, la producción de hidroenergía es la más barata con un costo de $\notin 15 / \mathrm{kW} / \mathrm{h}$, seguido por la geotérmica con $\notin 20 / \mathrm{kW} / \mathrm{h}$. La energía térmica que es la siguiente alternativa de mayor uso, no la siguiente más barata, tiene un costo de $ф 40.36 / \mathrm{kW} / \mathrm{h}$. La producción media de hidroenergía es de $0.53 \mathrm{~kW} / \mathrm{h} / \mathrm{m}^{3}$. Con base en lo anterior, y considerada la geotérmica como la siguiente mejor alternativa, se estimó que el valor del agua es de $\varnothing 2.67 / \mathrm{m}^{3}$. Mientras que si se considera la producción de energía térmica, la estimación del valor del agua sería de $\notin 13.56 / \mathrm{m}^{3}$. Para efectos de posibles ajustes en tarifas y cánones, el valor considerado es el de $\phi 2.67 / \mathrm{m}^{3}$.

\subsubsection{Valor del agua en el sector agropecuario}

Con relación al enfoque de cambio en productividad, su aplicación está asociada a que hay un reconocimiento de que el riego incrementa la productividad agrícola y este cambio en la producción puede ser usado para calcular el valor del agua. Este cambio en la producción multiplicado por el precio del producto agrícola (mercado) aproxima el valor del agua usada en agricultura. En ese sentido, aunque la productividad agrícola está en función de una serie de condiciones climáticas y agroecológicas; el agua es necesaria para que se realice el balance hídrico dentro de la planta y, el intercambio de nutrientes como parte del proceso de fotosíntesis.

La agricultura usa el agua en el riego de los cultivos, y es de las actividades que mayor consumo tiene del recurso. En condiciones normales, más del $80 \%$ del agua disponible se dedica a la agricultura (Middleton 1995). El riego incrementa la productividad agrícola y este cambio en la producción puede ser usado para calcular 
el valor del agua. Este cambio en la producción multiplicado por el precio del producto agrícola (mercado) aproxima el valor del agua usada en agricultura.

Al existir información sobre el efecto del riego en la productividad de los cultivos que lo utilizan, se puede estimar el valor del cambio en la productividad por el uso del agua. Por ejemplo, si el cultivo $\mathbf{k}$ experimenta un cambio en la producción cuando está bajo riego, entonces el valor del agua sería:

$$
\mathrm{P}_{\mathrm{k}}^{\mathrm{ag}}=\left(\mathrm{p}_{\mathrm{k}}-\mathrm{c}_{\mathrm{k}}\right) * \mathrm{q}_{\mathrm{k}}
$$

Además,

$$
\mathrm{q}_{\mathrm{k}}=\left(\mathrm{Q}_{\text {riego }}^{\mathrm{k}}-\mathrm{Q}_{\text {secano }}^{\mathrm{k}}\right) / \mathrm{V}_{\mathrm{i}}
$$

Donde,

$\mathrm{P}_{\mathrm{k}}{ }^{\mathrm{ag}} \quad$ Costo del agua en agricultura para el cultivo $\mathrm{k}(\phi / \mathrm{m} 3)$

$\mathrm{p}_{\mathrm{k}} \quad$ Precio del producto $\mathrm{k}(\phi / \mathrm{kg})$

$\mathrm{c}_{\mathrm{k}} \quad$ Costo de producción bajo riego $(\phi / \mathrm{kg})$

$\mathrm{q}_{\mathrm{k}} \quad$ Cambio en producción del cultivo $\mathrm{k}$ bajo riego $(\mathrm{kg} / \mathrm{m} 3)$

$\mathrm{Q}_{\text {riego }}^{\mathrm{k}} \quad$ Cantidad de producción del cultivo $\mathrm{k}$ bajo riego $(\mathrm{kg} / \mathrm{ha})$

$\mathrm{Q}_{\text {secano }}^{\mathrm{k}} \quad$ Cantidad de producción del cultivo $\mathrm{k}$ sin riego $(\mathrm{kg} / \mathrm{ha})$

$\mathrm{V}_{\mathrm{i}} \quad$ Volumen de agua usado en riego del cultivo i (m3/ha)

Si la información es para n cultivos, se puede calcular el valor del agua como un promedio ponderado (Pag) de los n cultivos analizados. Es decir,

$$
P^{a g}=\frac{\sum_{i=1}^{n} P_{i}^{a g} Q_{i}}{\sum_{i=1}^{n} Q_{i}}
$$

En la cuenca del Río Tempisque la gran parte de la actividad agropecuaria se da bajo riego debido a la escasez de agua y de una irregularidad en las precipitaciones. Para estimar el valor del agua se consideró la información disponible en el Distrito de Riego Arenal-Tempisque, para un conjunto de actividades agrícolas, y a los resultados en el estudio desarrollado por Castro y Barrantes (1998). El valor correspondiente asociado al uso del agua en el sector agropecuario es de $\not 2.89 / \mathrm{m}^{3}$. 


\subsubsection{Resumen del valor del agua como insumo de la producción}

Los valores estimados por sector se presentan en el Cuadro 2 y que son la base para un ajuste en el valor del agua en las tarifas y cánones por aprovechamiento de agua. Considerado el volumen diferenciado por sector, se estimó un costo promedio ponderado, cuyo resultado es de $₫ 3.31 / \mathrm{m}^{3}$. A pesar del valor promedio ponderado, se propone, como ajuste en tarifas y cánones, un valor diferenciado cuya base sean las estimaciones hechas en cada sector.

Cuadro 2. Valor de uso del agua como insumo de la producción para la cuenca del Río Tempisque

\begin{tabular}{ccc}
\hline Sector & $\begin{array}{c}\text { Demanda } \\
\text { mill } \mathrm{m}^{3} / \text { año }\end{array}$ & $\begin{array}{c}\text { Precio } \\
\phi / \mathrm{m}^{3}\end{array}$ \\
\hline Doméstico & 17,10 & 3.57 \\
Industrial & 185,17 & 13.67 \\
Agropecuario & $4,370,94$ & 2.89 \\
Hidroenergía & 191,74 & 2.67 \\
\hline Total & $4,764,94$ & 3.31 \\
\hline
\end{tabular}

Fuente: Elaboración propia

\subsection{Margen de ahorro-inversión}

El margen de ahorro-inversión es un componente en el ajuste de la tarifa hídrica cuyo fin es la de garantizar disponibilidad financiera para la atención de imprevistos que afecten el flujo del recurso hídrico hacia los diferentes sectores. Dichos imprevistos se relacionan con el daño a infraestructuras debido a inundaciones $u$ otros desastres, disminución crítica de la oferta hídrica por cualquier eventualidad, entre otras afectaciones. El margen de ahorro-inversión que se propone representa un 5\% del valor de captación, del de restauración y del de agua como insumo de la producción que finalmente se incorporen al ajuste de tarifas y cánones.

Al margen de la capacidad de pago de los distintos sectores, la estimación del costo del servicio ambiental hídrico indica que este recurso, dada la escasez en la región, es caro y que es necesario iniciar con el proceso de uso racional del mismo. Como no hay un reconocimiento de este servicio es posible considerar que hay un subsidio ambiental importante a las distintas actividades humanas que se desarrollan en la región. En caso de no implementarse las medidas necesarias, el costo social será mayor dado que se acrecentará la escasez hídrica, la infraestructura productiva podría colapsar y se podrían potenciar los procesos migratorios desde la región hacia otras regiones de mayor riqueza hídrica. 


\subsection{Valores para el ajuste ambiental en tarifas y cánones hídricos}

Las tarifas y cánones hídricos actuales, por lo general, contemplan los costos asociados al abastecimiento de agua fundamentalmente. No hay consideraciones ambientales en dichas tarifas, lo que justifica plantear el ajuste correspondiente, incorporar el valor de captación, el de restauración y el del agua como insumo de la producción. Además, se puede incorporar un componente asociado al margen de ahorro-inversión, que se propone en el estudio. De esta manera, se propone como base para el ajuste los valores estimados en el presente estudio y que están reflejados en el Cuadro 3.

Cuadro 3. Valores económicos en el servicio ambiental hídrico $\left(\varnothing / \mathrm{m}^{3}\right)$

\begin{tabular}{lccccc}
\hline Sector & $\begin{array}{c}\text { Valor de } \\
\text { captación }\end{array}$ & $\begin{array}{c}\text { Valor de } \\
\text { restauración de } \\
\text { bosques }\end{array}$ & $\begin{array}{c}\text { Valor del } \\
\text { agua como } \\
\text { insumo }\end{array}$ & $\begin{array}{c}\text { Margen } \\
\text { ahorro } \\
\text { inversión }\end{array}$ & Total \\
\hline Doméstico & 1.67 & 3.02 & & 0.23 & 4.92 \\
Industrial & 1.67 & 3.02 & 13.67 & 0.92 & 19.27 \\
Agropecuario & 1.67 & 3.02 & 2.89 & 0.38 & 7.95 \\
Hidroenergía & 1.67 & 3.02 & 2.67 & 0.37 & 7.72 \\
Otros & 1.67 & 3.02 & & 0.23 & 4.92 \\
\hline
\end{tabular}

Fuente: Elaboración propia

\section{Disposición de pago por el servicio ambiental hídrico en la cuenca del Río Tempisque}

Con el fin de analizar la viabilidad de éxito de una política de ajuste de tarifas y cánones incorporado el servicio ambiental hídrico, se realizó una encuesta de disposición de pago en la cuenca del Río Tempisque. Los resultados están con base en 375 encuestas en el sector residencial, con un error máximo de 5\%, y que se aplicaron con base en una selección muestral que tomó como unidad de análisis el segmento censal.

De acuerdo con los resultados del estudio, el $81 \%$ de la población está dispuesta a pagar para proteger bosques donde se encuentran las fuentes que abastecen de agua a la población de la cuenca del río Tempisque. El monto de la disposición a pagar, en promedio es de $₫ 621.38$ por encima de lo que de los $₫ 2295.29 /$ mes que actualmente se paga por el consumo de agua potable. Para esta disposición de pago se asocia un ingreso familiar promedio de $ф 123,086 /$ mes. De acuerdo al ingreso familiar y a la tarifa actual, la disposición de pago representa el $27.07 \%$ de la tarifa de agua potable y el $0.5 \%$ del ingreso familiar. 
Esta disposición de pago representa un monto de $₫ 26 / \mathrm{m}^{3}$ aproximadamente, dado que el nivel de consumo mensual es de aproximadamente $24 \mathrm{~m}^{3}$. Esta disposición de pago podría estar explicado dado el nivel de conciencia en la población por la conservación de bosque (81\%), mientras que el $91 \%$ señala que habrá problemas de escasez debido principalmente a deforestación y quemas.

\subsection{Disposición de pago en el sector industrial}

En el caso del sector productivo se hizo una encuesta telefónica a usuarios abastecidos por Acueductos y Alcantarillados (AyA), dado que es difícil en tiempo y costos, así como en acceso encuestar a este sector con visitas de campo. También, dado que la mayoría de los usuarios del sector reproductivo tienen concesiones dadas por el Departamento de Aguas del Ministerio de Ambiente y Energía (MINAE), no tienen información ni de la demanda real ni de los costos en que se incurre para mantener la concesión, por las mismas características de cómo se administran las concesiones.

Se aplicaron 48 encuestas en total distribuidas en los diferentes cantones atendidos por la institución. Los resultados indican que el 100\% está dispuesto a pagar para proteger bosques en relación con la conservación de agua. El monto promedio de la disposición a pagar es de $₫ 1,110.87 /$ mes mientras que la tarifa actual por el servicio que brinda AyA es de $\not 14,751.91 / \mathrm{mes}$, que es muy similar a los registros que actualmente tiene la institución ( $\$ 13926.05 / \mathrm{mes}$ ). Dado el consumo de agua equivalente a 35.44 $\mathrm{m}^{3} / \mathrm{mes}$ en AyA, el pago adicional es de $\not 31.35 / \mathrm{m}^{3}$.

De las consultas realizadas en el sector reproductivo, el 100\% considera importante conservar bosques; mientras que el $91.67 \%$ considera que habrá escasez de agua en el futuro, debido fundamentalmente a la deforestación (73.0\%) y el desperdicio. Por otro lado, el $77.08 \%$ de los consultados están satisfechos con el servicio de agua potable, la cantidad de agua (93.75\%) y la calidad de la misma (83.33\%). Estos porcentajes muestran el nivel de conciencia que tienen los consultados en relación con la problemática de la disponibilidad de agua en el futuro, así como también sobre la necesidad de conservar los bosques para tal fin. Un dato adicional se refiere a que el $91.67 \%$ de los consultados considera importante que la población tenga medidor de agua para controlar el consumo y mejorar la administración de la demanda.

La principal observación expuesta por los usuarios es la necesidad de que se garantice el uso de los fondos para fines de protección del bosque, dado que hay una desconfianza en las instituciones relacionada con el manejo de recursos financieros. Esta es una condicionante que obliga a la definición de una figura financiera y una administración de fondos que garanticen que los recursos se destinen para los fines que se cobrarían, y en los sitios donde se genera el servicio ambiental hídrico. 


\section{Análisis del valor propuesto para el ajuste de tarifas y la disposición de pago}

El valor total obtenido en el ajuste de tarifa (Cuadro 3) considerados los cuatro aspectos relacionados con el servicio ambiental hídrico, es inferior a la estimación de la disposición máxima de pago. Mientras que la disposición de pago en el sector residencial es de $\phi 26.44 / \mathrm{m}^{3}$, el monto de ajuste es de $\phi 4.92 / \mathrm{m}^{3}$ o sea más de cinco veces el valor estimado para el servicio ambiental hídrico. Por su parte, en el sector reproductivo la disposición de pago es de $₫ 31.35 / \mathrm{m}^{3}$, mientras que el ajuste máximo es de $\not 19.27 / \mathrm{m}^{3}$.

Como la diferencia entre la disposición de pago y el valor económico estimado es alta, se propone iniciar el proceso de cobro considerado el pago de los componentes identificados en el servicio ambiental hídrico. Para tal efecto, es necesario definir la política tarifaria concreta que permita la incorporación de tales componentes. Los componentes valor de captación y valor de restauración deben ser compensados por todos los usuarios del recurso hídrico. En cuanto al valor de captación se justifica por la urgente necesidad de conservar el bosque en la región Chorotega, particularmente en la cuenca del Río Tempisque. Con relación al valor de restauración se debe a que muchas de las áreas de importancia hídrica han sido degradadas y se hace urgente restaurarlas con el fin de minimizar el riesgo de escasez futura de agua.

\section{Estimación de ingresos basado en la estrategia de cobro del servicio ambiental hídrico}

La aplicación de una tarifa hídrica ambientalmente ajustada con los componentes discutidos con anterioridad, generaría ingresos económicos equivalentes a $₫ 39,990.53$ millones por año para sustentar las actividades relacionadas con la administración del recurso hídrico (Cuadro 4). De acuerdo con los componentes valorados y los montos estimados para cada uno de ellos, los ingresos que se generarían responderían apropiadamente a un concepto de desarrollo social y económico, y a la conservación de los recursos hídricos, y, en general, a la de los ecosistemas, debido al aporte de recursos financieros para desarrollar actividades en el corto, mediano y largo plazo en el plano económico, social y ambiental. 
Cuadro 4. Estimaciones de ingreso basada en la estrategia de cobro (millones de colones/año)

\begin{tabular}{lllllll}
\hline Sector & Demanda & $\begin{array}{l}\text { Valor } \\
\text { de captación }\end{array}$ & $\begin{array}{l}\text { Valor } \\
\text { de restauración }\end{array}$ & $\begin{array}{l}\text { Valor } \\
\text { del agua }\end{array}$ & $\begin{array}{l}\text { Ahorro } \\
\text {-inversión }\end{array}$ & Total \\
\hline Doméstico & 17.10 & 28.47 & 51.59 & & 4.00 & 84.07 \\
Industrial & 185.17 & 308.38 & 558.75 & $2,530.38$ & 169.88 & $3,567.38$ \\
Agropecuario & $4,370.94$ & $7,279.44$ & $13,189.64$ & $12,644.73$ & $1,655.69$ & $34,769.50$ \\
Hidroenergía & 191.74 & 319.33 & 578.59 & 512.54 & 70.52 & $1,480.98$ \\
Otros & 18.02 & 30.01 & 54.38 & & 4.22 & 88.61 \\
\hline Total & $4,782.96$ & $7,965.63$ & $14,432.94$ & $15,687.65$ & $1,904.31$ & $39,990.53$ \\
\hline
\end{tabular}

Nota: En el sector industrial está incluido el turismo, que hasta ahora no contempla una categoría específica para separarlo.

Fuente: Elaboración propia

Del Cuadro 4 se desprende un apoyo financiero importante para la conservación de los bosques disponibles en la Cuenca del Río Tempisque con un monto de $₫ 7,965.63$ millones anuales. Por su parte, para la restauración de las zonas degradadas se espera un ingreso de $ф 14,432.94$ millones anuales. Lo más interesante de estos componentes es que se pueden convertir en una fuente permanente, continua y creciente, lo que posibilita adquirir compromisos de largo plazo, respondiendo apropiadamente a un condicionante que establece la conservación de bosque: la disponibilidad financiera para apoyar actividades de largo plazo.

Con los ingresos obtenidos por el cobro del agua como insumo de la producción, se pueden sustentar financieramente actividades relacionadas con el desarrollo de infraestructura social, investigación y educación. Y por último, con los ingresos generados por el margen de ahorro-inversión se da contenido financiero a la posibilidad de dar respuestas y oportunidades al surgimiento de eventos inesperados tales como desastres naturales o crecimiento anormal de la población.

La conveniencia y relevancia de la tarifa hídrica ambientalmente ajustada es un compromiso inevitable para garantizar la conservación de los recursos hídricos y de los ecosistemas, así como también, garantizar una mayor seguridad de que el desarrollo económico y social no se vea estrujado por la disminución del recurso hídrico en cantidad y calidad. Al contrario, existe la posibilidad de que en el mediano y largo plazo, los costos del desarrollo tiendan a una disminución que provoque una competitividad mayor de las actividades económicas en el comercio mundial. 


\section{Análisis del monto pagable a propietarios de tierras de importancia hídrica}

Con respecto a los dueños de las tierras que serían consideradas para el pago del servicio ambiental hídrico, se tomó como primera referencia el costo de oportunidad de esas tierras, debido a la renuncia de los ingresos que le generaría la actividad económica que compite por el uso de esas tierras. La principal actividad identificada es la ganadería, principalmente extensiva con aproximadamente 0.8 unidades animal por hectárea.

Con base en el costo de oportunidad que sirvió de base para la estimación del valor de captación hídrica del bosque, se determina que el ingreso esperado para el propietario es de $\notin 27,960.40 /$ ha/año. Este monto es superior al monto de $ф 15,832 /$ ha/año, que actualmente se pagan por conservación de bosques en Costa Rica. Por otro lado, en un estudio realizado en Heredia se determina que el ingreso promedio esperado por dueños de la tierra es de $\notin 23,000 /$ ha/año aproximadamente; monto que es inferior al propuesto en el caso para la cuenca del Río Tempisque.

Es muy importante señalar que la diferencia dada entre el costo de oportunidad y el ingreso esperado por pago del servicio ambiental hídrico, sea compensada por los otros servicios que se empiecen a reconocer, tales como el de belleza del paisaje, biodiversidad y fijación de gases con efecto invernadero. De esta forma se garantizaría la conservación de la cobertura boscosa y de otros usos apropiados para la conservación de la biodiversidad. Además, se reconocería al propietario el valor total del bosque en función de los beneficios ambientales que brinda, lo que representa una situación de justicia en la distribución de costos y beneficios.

\section{Conclusiones}

- Hay una serie de amenazas que atentan contra la disponibilidad hídrica en la Cuenca del Río Tempisque. Por ejemplo, la deforestación o remoción de cobertura boscosa en sitios de importancia hídrica, reflejado en el $23 \%$ de la cuenca que se encuentra en conflicto de uso; la degradación de la cuenca por desarrollar actividades agropecuarias en suelos de aptitud forestal; y, la sobreexplotación del recurso hídrico más allá de un esquema de uso sostenible de los acuíferos y cuerpos de agua (la asignación de nuevas concesiones para el Río Tempisque están restringidas).

- De acuerdo con el presupuesto hídrico, en la Cuenca del Río Tempisque hay un déficit en agua superficial (377.51 mill. $\mathrm{m}^{3} / \mathrm{año}$ ) considerado lo que entra a la cuenca como oferta disponible por precipitación y otros medios $(5,086.02$ mill. $\left.\mathrm{m}^{3} / \mathrm{año}\right)$ y los aprovechamientos sociales inventariados $(5,908.40$ mill. $\mathrm{m}^{3} / \mathrm{año}$ ). Aquí hay que señalar que hay un aporte de $1,556.56 \mathrm{mill} . / \mathrm{m}^{3}$ desde el Distrito de Riego Arenal - Tempisque, lo que muestra que la Cuenca del Río 
Tempisque muestra altos niveles de sobreexplotación hídrica actualmente. Dado lo anterior, es probable que se explotan las reservas disponibles y esto podría conducir al agotamiento de acuíferos.

- A pesar de la escasez del recurso hídrico, no hay un valor económico que la refleje y, por lo tanto, las tarifas hídricas de los distintos servicios, incluido el canon de concesiones, no están ajustadas apropiadamente. Lo anterior implica que hay un subsidio ambiental en las distintas actividades productivas en las que se utiliza el agua, que resultara en ganancias extraordinarias para estos sectores. Esta falta de ajuste en las tarifas hídricas limita la capacidad institucional y las posibilidades de promover un uso sostenible del recurso, la protección adecuada de la cuenca y la restauración de zonas degradadas con importancia hídrica.

- Con base en la metodología empleada se determinaron los valores relacionados con el servicio ambiental hídrico en su distinta conceptualización:

- Un valor de captación hídrica equivalente a $₫ 1.67 / \mathrm{m}^{3}$, como reconocimiento al bosque por su función en la regulación del servicio hidrológico, tanto en cantidad como en calidad.

- Un valor de restauración de bosques equivalente a $₫ 3.02 / \mathrm{m}^{3}$, con el fin de aplicarlo en la recuperación de las zonas degradadas de importancia hídrica en la cuenca.

- Un valor del agua como insumo de la producción con base a las estimaciones hechas: $\$ 13.67 / \mathrm{m}^{3}$ en el sector industrial-turístico-comercial, $\phi 2.67 / \mathrm{m}^{3}$ en el sector agropecuario y $\notin 2.89 / \mathrm{m}^{3}$ en el sector de hidroenergía.

- Un valor de margen de ahorro - inversión equivalente $₫ 5 \%$ de los valores anteriores aplicable a cada sector para el soporte financiero de eventualidades de desastres.

- Al margen de lo anterior, la población y los distintos sectores reconocen la necesidad de iniciar un proceso de uso racional y sostenible del recurso en la Cuenca del Río Tempisque, dado que les podría afectar tanto a ellos como a las generaciones futuras. Reconocen que el bosque tiene una gran importancia para la conservación del recurso hídrico y están dispuestos a participar en las campañas de protección que se promuevan, así como a pagar un monto adicional en la tarifa para tal fin, que en el sector doméstico es de aproximadamente $₫ 621.30 /$ mes mientras que en sector productivo es de $ф 1110.87 / \mathrm{mes}$. Esta disposición de pago es superior a las estimaciones hechas para el cobro del servicio ambiental hídrico, por lo que parece razonable esperar que sea una política de precios exitosa en la región. La principal observación expuesta es la necesidad de que se garantice el uso de los fondos para fines de protección del bosque, dado que hay una desconfianza en las instituciones relacionada con el manejo de recursos financieros. 
- Los fondos que se recauden por el cobro del servicio ambiental hídrico en caso de que se ajusten ambientalmente tarifas y cánones para sustentar las siguientes actividades:

- Conservación de la cobertura actual de bosques a través del cobro del valor de captación, donde se propone que se destinen $₫ 27,960.40 /$ ha/año.

- Restauración de cobertura en zonas degradadas de importancia hídrica a través del valor de restauración, donde se propone un pago a propietarios de $ф 83,561.00 /$ ha/año.

- El desarrollo de obras de infraestructura social tales como embalses y plantas de tratamiento de agua, investigación y educación ambiental a través del valor del agua como insumo de la producción. En este caso habría que definir las proporciones de gasto correspondientes de acuerdo con criterios que definan las distintas autoridades.

- Establecer un fondo de contingencia para atender emergencias relacionadas con el recurso hídrico a nivel de oferta y demanda, a través del valor de 1 margen de ahorro - inversión.

\section{Referencias bibliográficas}

Agencia de Cooperación Internacional del Japón. 2002. Estudio del Proyecto de desarrollo rural de la cuenca media del río Tempisque. Informe de Progreso. Pacific Consultants Internacional Naigai Engineering Co. Ltd.

Álvarez D., Esteban. 1995. Impacto Hidrológico de la (De) Reforestación en las Regiones Tropicales. ISA, Dirección de Ecología y de Recursos Naturales, Medellín, Colombia.

Ander, E. 1991. El desafío ecológico. Editorial Universidad Estatal a Distancia UNED. San José, Costa Rica.

Azqueta Oyarzun, Diego. 1994. Valoración económica de la Calidad Ambiental. McGRAW-HILL/INTERAMERICANA DE ESPAÑA, S.A.

Azqueta, Diego; Ferreiro Antonio (Eds.). 1994. Análisis económico y gestión de recursos naturales. Alianza Editorial S.A., Madrid, España.

Barrantes, Gerardo y Vega Mauricio. 2002. Evaluación del servicio ambiental hídrico en la cuenca del río Tempisque y su aplicación al ajuste de tarifas. Preparado para ASOTEMPISQUE. Financiado por el Fondo Canje Deuda Costa Rica Canadá, el Programa de Pequeñas Donaciones (PPD) de PNUD y la Fundación CR-USA.

Bowers, 1997. Sustainability and Environmental Economics: An alternative text. Addison Wesley Longman Limited. 
Calvo, J. 1990. Water resource Development in Costa Rica 1970-2000. Hydrological Science Journal 35,2,4/1990.

Castro, E. y G. Barrantes (b). 1998. Valoración económico ecológico del recurso hídrico en la cuenca Arenal: El agua un flujo permanente de ingreso. Heredia, Costa Rica.

CATIE 1996. Costos de establecimiento y manejo de plantaciones forestales y sistemas agroforestales en Costa Rica. Turrialba, Costa Rica.

CCT/CINTERPEDS, (1995). Valoración Económico Ecológico del Agua: Primera aproximación para la interiorización de costos. Centro Científico Tropical (CCT)/ Centro Internacional en Política Económica para Centro América y el Caribe (CINTRPEDS) San José Costa Rica.

Heuveldop J. et al. 1986. Agroclimatología tropical. 1ª Ed. Editorial UNED. San José, Costa Rica.

Middleton, Richard. 1995. El agua limpia: un recurso frágil. Documento Verde \#4. Servicio Cultural e Informativo de los Estados Unidos de América. Versión en español por Felipe Ramírez García.

Ramakrishna, B. 1997. Estrategias de extensión para el manejo integrado de cuencas hidrográficas: conceptos y experiencias. Instituto Interamericano de cooperación para la agricultura. San José, Costa Rica.

Schosinsky, G. y M. Losilla. 2000. Modelo analítico para determinar la infiltración con base en la lluvia mensual. En la Revista Geológica de América Central. Escuela Centroamericana de Geología. Número 23, diciembre, 2000. Universidad de Costa Rica. Editorial de la Universidad de Costa Rica.

Reynolds, 1995. Las Aguas Subterráneas en Costa Rica: Un Recurso en Peligro. En: Utilización y manejo del recurso hídrico (Jenny Reynolds Vargas, Editora). Editorial Fundación UNA, Heredia, Costa Rica.

Reynolds, 1997. Evaluación de los Recursos Hídricos en Costa Rica: Disponibilidad y Utilización. Documento preparado para el Proyecto de Cuentas Ambientales, coordinado por el Centro Internacional de Políticas Económicas de la Universidad Nacional y el Centro Científico Tropical. Heredia, Costa Rica.

Rudas, Guillermo. 1995. Uso del Agua e Incentivos Económicos para la Conservación de Cuencas Hidrográficas. Bogotá, Colombia.

United Nations. 1993. Integrated Environmental and Economic Accounting: Handbook of National Accounting. 


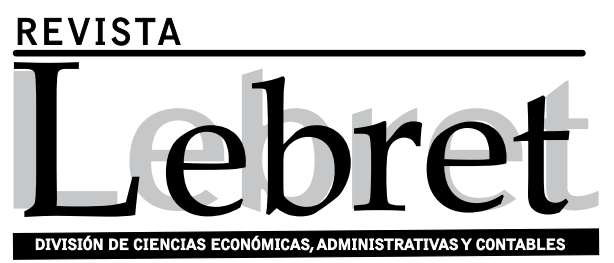

\section{CUPÓN DE SUSCRIPCIÓN}

\begin{tabular}{|l|}
\hline \multicolumn{1}{|c|}{ SUSCRIPTOR } \\
\hline Nombres \\
\hline Apellidos \\
\hline
\end{tabular}

\begin{tabular}{|c|c|c|}
\hline \multicolumn{3}{|c|}{ RESIDENCIA } \\
\hline \multicolumn{3}{|c|}{ Dirección } \\
\hline Ciudad & & País \\
\hline Telefono & fax & Celular \\
\hline
\end{tabular}

\begin{tabular}{|l|l|}
\hline & \multicolumn{2}{c|}{ SUSCRIPCIÓN ANUAL } \\
\hline Colombia $\$ 10.000$ & Exterior: US $\$ 5$ \\
\hline
\end{tabular}

\section{SUSCRIPCIÓN A PARTIR DEL NÚMERO}

\section{ESTIMADO SUSCRIPTOR}

- Consigne en la cuenta de ahorrros número 184-91-706-0 del Banco de Bogotá a nombre de Universidad Santo Tomás

- Envie copia del recibo de consignación, junto con este cupon a la División de Ciencias Económicas y Contables de la Universidad Santo Tomás en la carrera 18 № 9-27 Bucaramanga y copia a revistalebret@gmail.com 